\title{
Quantifying the Martian Geochemical Reservoirs: An Interdisciplinary Perspective
}

\author{
Michael J. Toplis · James F. Bell • Eric Chassefière • \\ Christophe Sotin · Tilman Spohn • Michel Blanc
}

Published online: 14 December 2012

(C) Springer Science+Business Media Dordrecht 2012

The planet Mars has fascinated humanity since antiquity, a fascination that persists to the present-day, fuelled by the enticing possibility that the conditions necessary for life may once have existed there, and perhaps continue to exist to the present day. Indeed, comparative planetology of terrestrial bodies large enough to retain an atmosphere is a key to understanding the formation of our own planet and the physical and chemical conditions that led to the emergence and the development of life. In more general terms, the study of rocky bodies of the solar system also provides a context for planetary formation as a whole, shedding light on the geochemical similarities and differences between the principal objects of the inner solar system, as well as the cores of the outer planets and many of their satellites.

However, it was not until the advent of space-based exploration in the 1960s that quantification of the geochemical characteristics of our planetary neighbours first became possible. Indeed, our knowledge of Mars changed dramatically when Mariner 4 took the first close-up pictures in July 1965, observing a highly cratered arid landscape. In 1976 the Viking orbiters and landers provided ground-breaking insight into surface morphology and the chemistry of the soil and atmosphere on Mars, data that also led to confirmation that the ShergottiteNakhlite-Chassignite group of meteorites (SNC) most probably have a martian origin. This

M.J. Toplis $(\varangle) \cdot$ M. Blanc

IRAP-OMP, Toulouse, France

e-mail: michael.toplis@irap.omp.eu

J.F. Bell

ASU, Tempe, AZ, USA

E. Chassefière

Univ. Paris 11, Orsay, France

C. Sotin

JPL, Pasadena, USA

T. Spohn

DLR, Berlin, Germany 
link between Mars and a group of rocks that may be studied with Earth-based analytical tools opens the possibility for precious detailed quantification of certain mineralogical and geochemical features of the red planet.

Over the last fifteen years, space-based exploration of the solar system has increased dramatically, with more and more sophisticated orbiters and landers being sent to Mars: the Sojourner rover (NASA, 1997), the Mars Exploration Rovers Spirit and Opportunity (NASA, 2004-present), the Phoenix lander (2008), the Mars Global Surveyor orbiter (NASA, 19972006), the Mars-Odyssey orbiter (NASA, 2001-present), the Mars Express mission (ESA, 2004-present), and the Mars Reconnaissance Orbiter (NASA, 2006-present). This intense period, rich in unprecedented scientific results, has led to immense progress in our perception of Mars and of its evolution over geological time. Thanks to the wide range of scientific instruments carried by these missions, the scientific community has access to more or less global coverage of surface morphology at $\sim 1$ to $10 \mathrm{~m} /$ pix scales, as well as information concerning the composition and mineralogy of the surface/subsurface. In addition, the scientific payloads of the MER rovers and the Phoenix lander provide highly detailed information at the selected landing sites, also providing the opportunity for geological context to be appreciated in the case of the MER rovers. In parallel to this accumulation of remote data, advances in numerical simulations and laboratory experiments also shed new light on the geochemical evolution of the planet Mars.

It was within this context that the ISSI-Europlanet Workshop entitled "Quantifying the Martian Geochemical Reservoirs" was organized. The workshop was held in Bern in April 2011 with the objective to create a diverse interdisciplinary forum composed of scientists directly involved in space-based exploration of the Martian surface, meteoriticists studying SNC meteorites, and planetary and/or Earth scientists simulating, numerically or experimentally, the physical and chemical processes occurring on or within Mars. Considerable time was dedicated to questions and discussion throughout the workshop and during this time the participants' diverse fields of expertise led to stimulating and thought-provoking exchanges. It is those cross-disciplinary discussions that form the basis of the papers that may be found here.

More than forty individuals have collaborated to provide this overview of current knowledge of the past and present martian geochemical reservoirs. In addition to the detailed description of data from Mars and the methods used to obtain them, many of the contributions also emphasize comparison with features on Earth, providing a perspective on the extent to which our knowledge of terrestrial systems influences interpretation of data from Mars. Almost all chapters also identify areas that would benefit from future work and measurements, providing a view of the short-term and long-term future of the study of Mars.

In detail, this special volume begins with a paper by Brasser, describing current thoughts on the formation of Mars, the reasons for its relatively small size, the composition of its building blocks and its accretion time-scale, highlighting several recent studies that conclude that Mars may in fact be a planetary embryo that never grew to be a fully-fledged planet. The second contribution by Mezger et al. is largely based on isotopic studies of the SNC meteorites, which can be used to constrain the extent and time-scales of planet-wide differentiation into a core, mantle, and crust. These results highlight the fact that, in contrast to the Earth, Mars appears to have preserved chemical heterogeneities produced during the first few tens of millions of years of its history, implying that vigorous whole-mantle convection has not homogenized the martian silicate interior.

The subsequent four contributions focus on specific geochemical reservoirs and how they have evolved over geological time. The paper by Grott et al. considers the mantle and magmatic crust, bringing together an impressively diverse range of data, assessing to what extent the morphological, mineralogical, and compositional features of the martian surface 
can be reconciled with numerical models of interior convection as constrained by laboratory measurements. Overall, a very satisfactory first-order picture emerges, dominated by global cooling of a stagnant-lid planet, although the authors identify a significant number of areas for future research to confirm our understanding of the internal structure of Mars and its evolution over time. In the next contribution, Lammer et al. treat the question of the Martian atmosphere, and its principal sinks and sources of volatile species, in particular $\mathrm{H}_{2} \mathrm{O}$ and $\mathrm{CO}_{2}$. This chapter highlights the difficulties in quantifying the pressure and composition of the atmosphere in the past, although a robust case is made for loss of the original proto-atmosphere due to the high EUV flux of the young Sun, and for minimal loss of volatiles to space over the last 4 billion years. On a related topic, Lasue et al. focus on the martian hydrosphere/cryosphere, considering the morphological evidence for the presence of water, both at the present time and in the past. Those data are combined with constraints from orbital and in-situ measurements to provide quantitative estimates of the amounts of water present and the global distribution and structure of icy and/or liquid water subsurface reservoirs. That contribution is complemented by a paper by Mousis et al. who explore the geochemical consequences of clathrate formation in the martian sub-surface, highlighting the possibility that formation/dissociation cycles of clathrates could have potentially important implications for short-term seasonal variations in the abundance of noble gases such as $\mathrm{Ar}, \mathrm{Kr}$, and $\mathrm{Xe}$, as well as the intriguing possible detections of methane that have been described over the last few years.

The last three contributions focus on secondary minerals produced by interaction of the magmatic crust and the atmosphere, providing critical markers of past conditions at the martian surface. The first of these, by Gaillard et al., considers sulphates and in more general terms the sulphur cycle on Mars, considering how sulphur is partitioned between the core, mantle, and crust and in what chemical forms sulphur is transferred to the atmosphere. This chapter highlights the fact that the iron-rich nature of the martian mantle has important consequences for the concentrations of sulphur that may be transported by magmatism, and that the fate of degassed sulphur is critically dependent on a combination of the oxidation state and water content of the mantle, and/or the pressure of the atmosphere, the transition to sulphate-dominated outcrops in the Hesperian being tentatively explained by a decrease in atmospheric pressure relative to earlier epochs. The contribution of Niles et al. focuses on carbonates, as seen in the SNC meteorites and as observed on the surface of Mars. This review quantifies the global concentration of carbonate and highlights the diversity of carbonate chemistry observed in martian rocks or outcrops, providing important constraints on the $\mathrm{CO}_{2}$ budget of Mars, the role of $\mathrm{Mg}$ - and Fe-rich fluids, and the interplay between carbonate formation and acidity. For example, despite outcrops that appear limited in spatial extent and primarily in terrains of Noachian age, evidence for active carbonate precipitation and the preservation of more ancient samples argues against global acidification of the martian surface during the Hesperian. The special issue ends with a contribution by Ehlmann et al. focussed on the presence of clays at the surface of Mars. This chapter reviews the chemical diversity and spatial distribution of clays, demonstrating that these volatile-bearing minerals are preferentially found in Noachian terrains, indicating a distinctive set of water-rock interaction processes during that time. However, different models of clay formation are considered, such as near-surface weathering, formation in ice-dominated near-surface ground waters, and formation by subsurface hydrothermal fluids. The extent of geochemical fractionation of the crustal reservoir and possible interactions with the atmosphere are discussed for each of these environments.

While each individual chapter in this volume provides a comprehensive look at a particular aspect of the geochemical evolution of Mars, taken as a whole, they offer a truly 
multidisciplinary look at the evolution of Mars over geological time, providing multiple but complementary viewpoints on numerous important issues. Of these, the question of the distribution of water over time is a case in point. For example, the contribution by Brasser underlines our lack of knowledge of the amount of water accreted to Mars during its growth, although Lammer et al., demonstrate that this uncertainty is mitigated to some extent by the fact that the primary atmosphere of Mars should have been rapidly lost to space. The contribution of Grott et al. highlights that the study of SNC meteorites and numerical models of convection both argue for water in the martian mantle, but that the amount could be anywhere from 2 to $200 \mathrm{ppm}$, providing few firm constraints on the impact of volcanic outgassing. Furthermore, Lammer et al., argue that it is extremely difficult to quantify the water content of the secondary atmosphere, not only due to uncertainties in the amount of water released during internal degassing, but also because the effect of meteoritic or cometary bombardment could either erode or add water to the atmosphere. In this respect, secondary minerals formed through interaction of magmatic rocks with the atmosphere/hydrosphere are important markers of surface conditions. Interestingly, all three contributions considering this approach (Gaillard et al., Niles et al., Ehlmann et al.) argue against the need for a 'warm and wet' early Mars, the case being made that the Noachian atmosphere was possibly of relatively low density and cold (though warmer and wetter than the current Mars atmosphere). Liquid water is proposed to have been present near the surface of Mars at certain times during the Noachian, but it is suggested that this could have occurred during transient periods of warmer conditions, possibly related to volcanism. On the other hand, Lasue et al., illustrate the diverse geomorphological evidence for significant amounts of water in the geological past, such as the spectacular late Hesperian outflow channels. However, they find no current evidence in the near subsurface for a corresponding large icy or liquid water reservoir, despite the fact that Lammer et al. conclude that if abundant water were present on Mars at some time after the Noachian, then it must still be there, as atmospheric loss processes to space are not sufficient to explain any more than a modest reduction in atmospheric pressure. These considerations thus beg the question of just how much water was present at the end the Hesperian, and where and in what form that water has been sequestered.

The critical issue of water on Mars thus appears far from being resolved, leaving ample room for future studies to provide additional constraints. In this respect, the successful arrival of the next generation rover Mars Science Laboratory (MSL, "Curiosity") in August 2012 will provide further key observations concerning the chemical and mineralogical diversity of ancient sedimentary rocks in Gale crater, hopefully providing new insight into the relations between magmatic precursors and the formation of carbonates, clays and sulphates. In the near future, the orbiter MAVEN (that will be launched in November 2013) will provide new data on the composition and dynamical state of the martian atmosphere and solar wind interactions, while critical information on the interior structure will be revealed by the seismic observations and heat-flow measurements to be made by the recently selected NASA InSight Discovery mission. Future missions like ESA's planned ExoMars rover or other future Mars orbiters, landers, and rovers will no doubt help to address and test the kinds of questions and hypotheses described in this volume.

Our understanding of Mars based on more than four decades of incredible discovery thus continues to evolve, and it is our hope that this collection of chapters will constitute a timely perspective on current knowledge and thinking concerning the geochemical evolution of Mars, providing context and a valuable reference point for even more exciting future discoveries. 


\section{References}

R. Brasser, The formation of Mars: building blocks and accretion time scale. Space Sci. Rev. (2012, this issue). doi:10.1007/s11214-012-9904-2

B. Ehlmann et al., Geochemical consequences of widespread clay mineral formation in Mars' ancient crust. Space Sci. Rev. (2012, this issue). doi:10.1007/s11214-012-9930-0

F. Gaillard et al., Geochemical reservoirs and timing of sulfur cycling on Mars. Space Sci. Rev. (2012, this issue). doi:10.1007/s11214-012-9947-4

M. Grott et al., Long-term evolution of the martian crust-mantle system. Space Sci. Rev. (2012, this issue). doi:10.1007/s11214-012-9948-3

H. Lammer et al., Outgassing history and escape of the martian atmosphere and water inventory. Space Sci. Rev. (2012, this issue). doi:10.1007/s11214-012-9943-8

J. Lasue et al., Quantitative assessments of the martian hydrosphere. Space Sci. Rev. (2012, this issue). doi: 10.1007/s11214-012-9946-5

K. Mezger et al., Core formation and mantle differentiation on Mars. Space Sci. Rev. (2012, this issue). doi: $10.1007 / \mathrm{s} 11214-012-9935-8$

O. Mousis et al., Volatile trapping in martian clathrates. Space Sci. Rev. (2012, this issue). doi:10.1007/ s11214-012-9942-9

P. Niles et al., Geochemistry of carbonates on Mars: implications for climate history and nature of aqueous environments. Space Sci. Rev. (2012, this issue). doi:10.1007/s11214-012-9940-y 